\title{
Highly efficient protocol for callogenesis, somagenesis and regeneration of Indica rice plants
}

\begin{abstract}
In the present study, we have reported a simple, fast and efficient regeneration protocol using mature embryos as explants, and discovered its effective applicability to a range of Indica rice genotypes. We have considered the response of six varieties in the steps of the regeneration procedure. The results showed that calli were variably developed from the scutellar region of seeds and visible within 6 ï 20 days. The highest and lowest calli induction frequency (70\% and 51.66\%) and number of induced calli from seeds (14 and 10.33) were observed in MR269 and MRQ74, respectively. The maximum and minimum number (7.66 and 4) and frequency of embryogenic calli (38.33\% and 20\%) were recorded in MR219 and MRQ74, respectively. However, the highest browning rate was observed in MR84 (87\%) and the lowest rate in MRQ50 (46\%). The majority of plants regenerated from embryogenic calli were obtained from MRQ50 (54\%) and the minimum number of plants from MR84. In this study, the maximum numbers of plantlets were regenerated from the varieties with highest rate of embryogenic calli. Also, various varieties, including MRQ50, MR269, MR276 and MR219, were satisfactorily responding, while MRQ74 and MR84 weakly responded to the procedure. Such a simple, successful and generalized method possesses the potential to become an important tool for crop improvement and functional studies of genes in rice as a model monocot plant.
\end{abstract}

Keyword: Indica rice; Genetic transformation; Regeneration potential; Mature embryo 